\title{
Genetic and Environmental Influences on General Skin Traits: Healthy Twins and Families in Korea
}

\author{
Young Ju Suh, ${ }^{1}$ Jeonghyun Shin, ${ }^{2}$ Moonil Kang, ${ }^{3}$ Hyun Ju Park, ${ }^{4}$ Kayoung Lee, ${ }^{5}$ Yun-Mi Song, ${ }^{6}$ \\ and Joohon Sung ${ }^{3,7}$ \\ ${ }^{1}$ Department of Biomedical Sciences, College of Medicine, Inha University, Incheon, Republic of Korea \\ ${ }^{2}$ Department of Dermatology, College of Medicine, Inha University, Incheon, Republic of Korea \\ ${ }^{3}$ Department of Epidemiology, School of Public Health, Seoul National University, Seoul, Republic of Korea \\ ${ }^{4}$ Department of Biostatistics, College of Medicine, Yonsei University, Seoul, Republic of Korea \\ ${ }^{5}$ Department of Family Medicine, Busan Paik Hospital, Inje University, College of Medicine, Busan, Republic of Korea \\ ${ }^{6}$ Department of Family Medicine, Samsung Medical Center, Sungkyunkwan University School of Medicine, Seoul, Republic \\ of Korea \\ ${ }^{7}$ Institute of Health and Environment, Seoul National University, Seoul, Republic of Korea
}

\begin{abstract}
Family study can provide estimates of overall genetic influences on a particular trait because family relationships provide accurate measures of average genetic sharing. However, evidence of genetic contributions to skin phenotypes is limited, which may preclude genetic studies to identify genetic variants or to understand underlying molecular biology of skin traits. This study aimed to estimate genetic and environmental contributions to selected dermatologic phenotypes, that is, to melanin index, sebum secretion, and skin humidity level in a Korean twin-family cohort. We investigated more than 2,000 individuals from 486 families, including 388 monozygotic twin pairs and 82 dizygotic twin pairs. Variance component method was used to estimate genetic influences in terms of heritability. Heritability of skin melanin index, sebum secretion, and skin humidity (arm and cheek) were estimated to be 0.44 [95\% Cl $0.38-0.49], 0.21$ [95\% Cl $0.16-0.26$ ], 0.13 [95\% $\mathrm{Cl} 0.07-0.18$ ], and 0.11 [95\% $\mathrm{Cl} 0.06-0.16$ ] respectively, after adjusting for confounding factors. Our findings suggest that genetics play a major role on skin melanin index, but only mild roles on sebum secretion and humidity. Sebum secretion and skin humidity are controlled predominantly by environmental factors notably on shared environments among family members. We expect that our findings add insight to determinants of common dermatologic traits, and serve as a reference for biologic studies.
\end{abstract}

Keywords: melanin index, sebum secretion, skin humidity, twins, heritability

Paying attention to one's appearance is a part of basic human behavior, with which many daily life decisions are made. Many skin traits are related to appearance, and skin color is probably one of the most distinctive traits that characterize complexion.

Skin color is affected by melanin, carotenoids, oxyhemoglobin, and hemoglobin itself or its metabolite such as bilirubin (Freedberg et al., 2003). In addition, clinical conditions, such as jaundice, flushing, anemia, and melanin level affected by sunlight play important roles in determining complexion. Many people have been very interested in skin pigmentation disorders such as melasma and vitiligo. Dermal hyperpigmentary diseases are commonly found in Asians (Kang, 2012). Genetic factors have been reported to play significant roles in skin pigmentation, with heritabilities $\left(\mathrm{h}^{2}\right)$ ranging from 0.34 to 0.90 (Byard, 1981; Clark et al., 1981; Paik et al., 2012). The importance of genetic influence on melanin index response to sunlight exposure has also been reported (Barsh, 2003).

Levels of sebum secretion and skin humidity are additional traits that influence dermatologic conditions and aesthetic characteristics. Individual variations in sebum and humidity levels may be associated with the risk of acne or eczematous skin lesions. Sebum is a secretion of liquefied fat from sebaceous glands and protects the skin surface from drying. Alterations in sebum secretion are

RECEIVED 5 April 2016; ACCEPTEd 22 August 2016. First published online 16 November 2016.

ADDRESS FOR CORRESPONDENCE: Joohon Sung, Department of Epidemiology, School of Public Health, Seoul National University, 559 Gwanak-ro, Gwanak-gu, Seoul, 08826, Republic of Korea.E-mail: jsung@snu.ac.kr 
associated with common dermatologic conditions, such as acne vulgaris and seborrheic dermatitis, and a sebum excretion rate correlates with the severity of acne (Bataille et al., 2012; Bataille et al., 2002; Walton et al., 1988). The heritability estimate of acne was reported as 0.81 in the UK Twin Registry. Prevalence of acne is found in $70 \%$ of adolescents in Korea (Cho et al., 2014). The study reported that family history of acne is associated with increased risk of occurrence of acne in Korea (Cho et al., 2014). Sebum excretion is considered to be under genetic control but modified by environmental factors (Walton et al., 1988). Skin humidity is maintained by the skin barrier function but influenced by exogenous factors such as climate and endogenous factors such as hormones (Pons-Guiraud, 2007). Dysregulation of skin humidity is associated with xerosis, pruritus, increased irritability, and aggravation of existing skin diseases such as atopic dermatitis, psoriasis, hand eczema, contact dermatitis, or xerotic eczema (Miyagaki \& Sugaya, 2015). However, to our knowledge, it has not been reported to what extent genes are involved in the regulation of skin humidity. Inference from indirect evidence might suggest the role of genetics in skin humidity control; that is, a recent metaanalysis of genome-wide association studies found that mutation in the filaggrin gene, known to stabilize the skin barrier (Ginger et al., 2005), is associated with atopic dermatitis, in which humidity may modify the onset or severity of the disease (Paternoster et al., 2012).

Heritability, which is a proportion of additive genetic variation out of total phenotypic variation, commonly evaluates the genetic contributions to the traits. The rest of the variance is explained by shared family environment and unique environmental factors (Bataille et al., 2012). Because family relationships provide accurate measures of average genetic sharing, family studies can provide estimates of overall genetic influences on a particular trait. However, to our knowledge, evidence of genetic contributions to skin phenotypes is limited, which may preclude genetic studies to identify genetic variants or to understand underlying molecular biology of skin traits.

Family studies confer an advantage in dissecting genes and environments, and adding twins can enrich family studies. The presence of monozygotic (MZ) twins enables the discrimination of polygenic and shared environmental variances, and potentially increases the power of familybased studies (Abecasis et al., 2002; Bataille et al., 2012) In order to estimate genetic and environmental contributions to skin phenotypes and to identify associated risk factors, we measured skin melanin index, sebum secretion, and skin humidity levels by using standardized measures in a Korean twin-family cohort.

\section{Materials and Methods}

\section{Participants}

The subjects involved in this study were selected from the Korean Healthy Twin Study, which is a twin-family cohort study aimed at investigating the genetic and environmental risk factors associated with complex diseases. The study cohort consisted of same-sex twin pairs ( $\geq 30$ years old) and their first-degree adult family members. Participants were recruited by mail, based on the existing nationwide twin-family register, and by advertisement. The study design for the Korean Healthy Twin study has been previously described in detail (Gombojav et al., 2013; Sung et al., 2006). The skin survey was accompanied with the general health examination, which requires fasting for 12 hours (for glucose and lipids test). A very limited amount of water is allowed but no alcohol. Epidemiologic and clinical data were recorded by qualified interviewers at two clinical centers of general hospitals at the Samsung Medical Center in Seoul, and at the Busan Paik Hospital in Busan from November 2005 to January 2011. The study protocol of the Healthy Twin Study was approved by the institutional review board (IRB) of each hospital. The declaration of Helsinki protocols was followed and participants in the Healthy Twin Study gave their written, informed consent.

We used 16 short tandem repeat markers (AmpFISTR Identifier Kit; PerkinElmer, Norwalk, CT) to confirm the zygosity until 2009, and then a zygosity questionnaire validated for accuracy (Song et al., 2010). Family relationships were further confirmed using 1 million SNP markers (Affymetrix GeneChip version 6).

\section{Measurements}

Skin phenotypes were measured by using a $\mathrm{C}+\mathrm{K}$ multi probe adapter MPA 9 and probes (Courage + Khazaka Electronic GmbH, Köln, Germany). A Mexameter ${ }^{\circledR}$ MX 18 was used to assess melanin indices (MIs), as described by the EEMCO (European Group for Efficacy Measurement on Cosmetics and Other Topical Products; Pierard, 1998). The probe type used produces light and a receiver measures light reflected by skin. The MI value is computed as the ratio of the quantity of light emitted by the probe and the amount absorbed by the skin. Skin MIs were measured at three sites, that is, a cheek, the flexor surface of the right upper arm, and the lumbar area of the back. The authors selected the back region because it is less affected by exposure to sunlight. The participants were instructed to avoid applications of facial or body cream for 1 day before coming for their health examination and survey. Measurements of sebum secretion were taken from the glabella after washing the face for 30 minutes with a standardized detergent, using a Sebumeter ${ }^{R}$ SM 815, as described by the EEMCO (Pierard et al., 2000). For skin humidity, measurements were taken from two sites, on a cheek and on the flexor surface of the right upper arm. Hydration level was estimated by using a Corneometer ${ }^{B}$ CM825, as described by the EEMCO (Berardesca, 1997). All skin measurements were repeated three times, and if two measurements differed 
by more than $10 \%$, the median value of the three were used, otherwise the average was taken; room temperature was maintained at $18-23^{\circ} \mathrm{C}$ and a relative humidity of 40-60\%.

\section{Statistical Analysis}

We explored the relationships between continuous skin phenotypic variables (melanin index, sebum, and humidity) and covariates such as, age, sex, serum creatine, medical histories (hyperthyroidism, diabetes mellitus, atopic dermatitis, and chronic hepatitis/liver cirrhosis), smoking status, and the season when measurements were taken, using Spearman correlation analysis (for continuous variables), Wilcoxon rank sum test (Mann-Whitney $U$-test for dichotomous variables), or the Kruskal-Wallis test (for categorical variables). Statistical analysis was performed by using SAS version 9.3 (SAS Institute, Cary, NC, USA). Intraclass correlation coefficients (ICC) of skin phenotypes were estimated to evaluate familial correlations between specific family pairs in the pedigree by using S.A.G.E. software, version 6.1 (http://darwin.cwru.edu/sage/). Heritabilities $\left(\mathrm{h}^{2}\right)$ of each skin phenotype were estimated by using variance components methods using the SOLAR-Eclipse software package (http://solar-eclipse-genetics.org/). For heritability analysis, rank-based inverse normal transformation was applied to sebum and humidity values with deviated nonnormal distributions (Beasley et al., 2009). Estimated heritabilities of skin phenotypes were adjusted for linear and non-linear effects of age, sex, the interaction between age and sex, and other covariates found to be significant. Differences between twins and non-twins are dissected into their respective genetic distances in the analysis. Identical twins show $100 \%$ genetic correlations, whereas fraternal twins shares $50 \%$ of genetics (the same as a usual sibling). Being a same family are further considered as random effects (as shared familial environments) in the model. The bestfitting model was selected based on likelihood estimates, for different models explained by additive genetics (A), nonadditive genetics (= dominant genetics, D), unmeasured shared environments (C), and unique environments (E). Shared environmental components with household effects were considered by using the ACE model (including additive genetics and unmeasured shared and unique environments in the variance component model) for each skin phenotype.

\section{Results}

Initially, 3,079 subjects from 661 families, with $531 \mathrm{MZ}$ and 121 dizygotic (DZ) twins were eligible for this study, and of these, 2,089 individuals (484 families, $385 \mathrm{MZ} / 81$ DZ) were included in the analyses for melanin index, 2,167 (476 families, $375 \mathrm{MZ} / 81 \mathrm{DZ}$ ) for sebum production, and 2,185 (486 families, $388 \mathrm{MZ} / 82 \mathrm{DZ}$ ) for skin humidity.
Table 1 shows the distribution of subject characteristics in relation to skin phenotypes. Subject age was significantly correlated with each skin phenotype $(r=-0.214$, $p$ value $<.0001$ for melanin index, $r=-0.061, p$ value $=.005$ for sebum, $r=0.043$, $p$ value $=.044$ for humidity in arms, and $r=0.066, p$ value $=.002$ for humidity in cheeks by Spearman correlation analysis). Comparing the ranks of melanin index phenotype for other covariates, they were found to differ by history of diabetes mellitus, atopic dermatitis ( $p$ values $<.05$ by Wilcoxon rank sum test), and smoking status ( $p$ value $<.0001$ by the Kruskal-Wallis test). Ranks of sebum differed by sex ( $p$ value $<.0001$ by Wilcoxon rank sum test), smoking status, and season $(p$ value $<.0001$ by the Kruskal-Wallis test), and were significantly correlated with creatine $(r=0.244, p$ value $<.0001$ by Spearman correlation analysis). When we compared the MZ ICC for melatonin indices at different sites, ICC $=0.745[95 \%$ $\mathrm{CI}=0.704-0.780]$ at cheek, followed by the ICC $=0.705$ [95\% CI $=0.657-0.744]$ at the flexor surface of right upper arm, and ICC $=0.593$ [95\% CI $=0.548-0.638]$ at the lumbar area of the back. Comparing the ranks of arm humidity for each covariate, they were found to differ by history of atopic dermatitis ( $p$ value $<.05$ by Wilcoxon rank sum test), and season ( $p$ value $<.0001$ by the Kruskal-Wallis test). The ranks of cheek humidity differed by sex, history of hyperthyroidism ( $p$ value $<.05$ by Wilcoxon rank sum test), smoking status, and tested season ( $p$ value $<.05$ by the Kruskal-Wallis test), and were negatively correlated with creatine $(r=-0.103, p$ value $<.0001$ by Spearman correlation analysis).

Supplementary Table S1 presents the association between clinical characteristics and each skin phenotype. Except for age and smoking status, melanin index did not show significant associations. For sebum secretion index and skin humidity, age, sex, seasonal variation, and medical history (chronic hepatitis/liver cirrhosis, atopic dermatitis, or hyperthyroidism) showed associations (Supplementary material is available on the Cambridge Journals Online website.).

Table 2 shows the familial correlations between possible pair of family members for each skin phenotype. Correlations between MZ twins were moderately significant with $r=0.593$ for melanin index, $r=0.459$ for sebum, $r=0.379$ for arm humidity, and $r=0.460$ for cheek humidity (all $p$ values $<.0001$ ), and these were higher than between pairs of full siblings $(r=0.315$ for melanin index, $r=0.242$ for sebum, $r=0.249$ for arm humidity, and $r=0.315$ for cheek humidity, all $p$ values $<.0001)$ and that between parentoffspring pairs $(r=0.222$ for melanin index, $r=0.216$ for sebum, $r=0.232$ for arm humidity, and $r=0.298$ for cheek humidity, all $p$ values $<.0001)$. Spousal correlation estimates of sebum and humidity were significant with $r=$ 0.203 for sebum ( $p$ value $=.0012$ ), $r=0.240$ for arm humidity $(p$ value $=.0001)$, and $r=0.373$ for cheek humidity $(p$ value $<.0001)$ 
TABLE 1

Distribution of Study Population Characteristics in Relation to the Skin Phenotypes

\begin{tabular}{|c|c|c|c|c|c|c|c|c|c|}
\hline & \multirow[b]{2}{*}{$\begin{array}{l}N(\%) \text { or } \\
\text { Median }(I Q R)^{a}\end{array}$} & \multicolumn{2}{|c|}{$\begin{array}{l}\text { Melanin index } \\
(N=2,089)\end{array}$} & \multicolumn{2}{|c|}{$\begin{array}{l}\text { Sebum } \\
(N=2,167)\end{array}$} & \multicolumn{2}{|c|}{$\begin{array}{l}\text { Arm humidity } \\
(N=2,185)\end{array}$} & \multicolumn{2}{|c|}{$\begin{array}{l}\text { Cheek humidity } \\
\qquad(N=2,185)\end{array}$} \\
\hline & & $p$ & $p$ value ${ }^{b}$ & $\begin{array}{l}\text { Median } \\
\text { (IQR) }\end{array}$ & $p$ value & $\begin{array}{l}\text { Median } \\
\text { (IQR) }\end{array}$ & $p$ value & $\begin{array}{l}\text { Median } \\
\text { (IQR) }\end{array}$ & $p$ value \\
\hline Age (year) & $41(34-54)$ & $132(102-165)$ & $\begin{array}{l}<.0001 \\
(-.214)^{c}\end{array}$ & 56 (28-99) & $\begin{array}{l}.005 \\
(-.061)\end{array}$ & $39.2(31.7-47.9)$ & $\begin{array}{l}.044 \\
(.043)\end{array}$ & $44.9(29.8-57.5)$ & $\begin{array}{l}.002 \\
(.066)\end{array}$ \\
\hline \multicolumn{10}{|l|}{ Sex } \\
\hline Male & $1,377(42.3)$ & $167(100-167)$ & .857 & $85(47-145)$ & $<.0001$ & $39.0(30.9-47.6)$ & .214 & $40.3(27.4-53.4)$ & $<.0001$ \\
\hline Female & $1,879(57.7)$ & $132(103-164)$ & & $43(21-78)$ & & $39.3(32.0-48.3)$ & & $47.4(32.6-60.4)$ & \\
\hline Creatine & $0.9(0.8-1.0)$ & $132(102-165)$ & $\begin{array}{l}.226 \\
(-.027)^{c}\end{array}$ & $56(28-99)$ & $\begin{array}{l}<.0001 \\
(.244)\end{array}$ & $39.2(31.7-47.9)$ & $\begin{array}{l}.854 \\
(-.004)\end{array}$ & $44.9(29.8-57.5)$ & $\begin{array}{l}<.0001 \\
(-.103)\end{array}$ \\
\hline \multicolumn{2}{|c|}{ History of hyperthyroidism } & & .234 & & .006 & & .846 & & .025 \\
\hline Yes & $50(1.6)$ & $153(95-194)$ & & $30.5(16.0-64.0)$ & & $38.7(33.0-46.0)$ & & $54.1(41.5-63.5)$ & \\
\hline No & $3,029(98.4)$ & $132(102-165)$ & & 57 (29-99) & & $39.2(31.7-48.0)$ & & $44.7(29.8-57.4)$ & \\
\hline \multicolumn{2}{|c|}{ History of diabetes mellitus } & & .0001 & & .903 & & .797 & & .994 \\
\hline Yes & $201(6.5)$ & $121(91-150)$ & & $62(26-105)$ & & $39.6(32.2-48.6)$ & & $43.3(31.0-57.6)$ & \\
\hline No & $2,878(93.5)$ & $133(103-167)$ & & $56(28-98)$ & & $39.2(31.7-47.9)$ & & $45.0(29.7-57.4)$ & \\
\hline \multicolumn{2}{|c|}{ History of atopic dermatitis } & & .033 & & .215 & & .011 & & .544 \\
\hline Yes & $151(4.9)$ & $121(92-156)$ & & $61(33-98)$ & & $36.7(31.2-43.5)$ & & $44.5(34.9-56.2)$ & \\
\hline No & $2,928(95.1)$ & $133(103-166)$ & & $56(28-99)$ & & $39.4(31.7-48.3)$ & & $44.9(29.5-57.5)$ & \\
\hline \multicolumn{3}{|c|}{ History of chronic hepatitis/liver cirrhosis } & .132 & & .927 & & 0.387 & & .648 \\
\hline Yes & $121(3.9)$ & $128(92-161)$ & & $56(26.5-99.5)$ & & $40.0(32.9-49.7)$ & & $42.5(31.0-54.3)$ & \\
\hline No & $2,958(96.1)$ & $132(102-166)$ & & 56 (28-99) & & $39.2(31.7-47.9)$ & & $45.0(29.8-57.5)$ & \\
\hline \multicolumn{2}{|c|}{ Smoking status } & & $<.0001$ & & $<.0001$ & & 0.556 & & .005 \\
\hline Current & $603(19.6)$ & $146(111-186)$ & & $71(37-133)$ & & $39.3(32.0-47.9)$ & & $42.9(29.5-54.8)$ & \\
\hline Former & 417 (13.5) & $128(96-159)$ & & $78(35-140)$ & & $39.2(31.5-46.2)$ & & $42.9(28.5-55.5)$ & \\
\hline Never & $2,059(66.9)$ & $130(101-162)$ & & $50(24-90)$ & & $39.2(31.7-48.5)$ & & $46.2(30.5-58.6)$ & \\
\hline \multicolumn{2}{|l|}{ Season } & & & & $<.0001$ & & $<.0001$ & & $<.0001$ \\
\hline Summer & $517(21.7)$ & & & $58(28-103)$ & & $43.1(35.1-52.2)$ & & $50.7(30.5-62.6)$ & \\
\hline Spring/fall & $1,032(43.3)$ & & & $64(31-119)$ & & $39.8(31.9-48.1)$ & & $46.0(29.5-59.6)$ & \\
\hline Winter & $835(35.0)$ & & & $49(24-84)$ & & $36.5(29.7-44.4)$ & & $42.0(30.0-52.1)$ & \\
\hline
\end{tabular}

The ADE model (including additive and dominant genetics and unique environments in the variance component model) for melanin index fitted better than the $\mathrm{AE}$ (including additive genetics and unique environments in the variance component model) or ACE models. For sebum and humidity phenotypes, the ACE model fitted better than the AE or ADE models; however, heritability estimates according to the ACE model tended to be lower than those of the AE or ADE models (Table 3). Heritability estimates for the AE model were $\mathrm{h}^{2}=0.547\left(\mathrm{~h}^{2}=0.573\right.$ adjusted for age, sex, age ${ }^{2}$, history of diabetes mellitus, atopic dermatitis, and smoking status) for melanin index, $\mathrm{h}^{2}=0.457\left(\mathrm{~h}^{2}=0.410\right.$ adjusted for age, sex, age $^{2}$, age ${ }^{2} \times$ sex, history of atopic dermatitis, smoking status, and season) for sebum, $\mathrm{h}^{2}=0.398$ $\left(\mathrm{h}^{2}=0.381\right.$ adjusted for age, histories of atopic dermatitis, and season) for arm humidity, and $\mathrm{h}^{2}=0.474\left(\mathrm{~h}^{2}=0.483\right.$ adjusted for age, sex, history of hyperthyroidism, smoking status, and tested season) for check humidity (all $p$ values $<$ $.0001)$. Heritability using the ACE model was significantly estimated as $\mathrm{h}^{2}=0.437$ (adjusted $\mathrm{h}^{2}=0.486$ ) for melanin index, $h^{2}=0.339$ (adjusted $h^{2}=0.211$ ) for sebum, $h^{2}=$ 0.147 (adjusted $\mathrm{h}^{2}=0.125$ ) for arm humidity, and $\mathrm{h}^{2}=$ 0.109 (adjusted $\mathrm{h}^{2}=0.109$ ) for cheek humidity (all $p$ values $<.05)$. Heritability using the ADE model was $\mathrm{h}^{2}=0.333$ (adjusted $\mathrm{h}^{2}=0.438$ ) for melanin index.

\section{Discussion}

Skin phenotypes, such as melanin index, sebum, and humidity are common complex traits that are explained by genetic and environmental factors. However, only a few genetic studies have addressed these skin phenotypes. Family studies of quantitative skin phenotypes enable the dissection of individual variation in skin phenotypes into genetic and environmental contributions, comparing the similarities of traits across different genetic relationship, assuming that they share certain level of common environments. In this study based on Korean healthy twins and their family members, each skin phenotype was found to be influenced by genetic and environmental components to varying degrees. Our heritability estimation finding suggests that over $43 \%$ of variation in skin melanin index in Korean twins and their families was conferred by genetic influences. Genetic studies on skin complexion involving genetically homogenous population have reported similar or larger genetic effects; $0.34 \sim 0.48$ for Australian (Clark et al., 1981) or 0.83 for Mongolian (Paik et al., 2012). Differences in study design and analytic methods make comparisons between previous studies and ours inconclusive. The study by Clark et al. (1981) measured pigmentation on more exposed skin areas and only involved twins. Thus, the findings from this study may reflect both melanin index and response to 
ultraviolet exposures. Additionally, involving only twins may inflate the heritability estimation, because nonadditive genetic effects are not easily discriminated from the additive genetic effects.

A study in a Mongolian population that measured skin darkness on the buttocks showed a very high level of genetic contribution of 0.83 (Paik et al., 2012). However, the participants in the study were younger, with an average age of 30 years, and shared environmental effects, including other environmental factors such as smoking and medical history of atopic dermatitis, were not considered. Considering that these environmental contributions were not accounted for in the Mongolian study, differences in the heritability estimates might be smaller with our study. We believe estimates in our study are stable because we involved more subjects with diverse genetic distances, and we accounted for a range of environmental factors.

We found that the heritability of sebum or humidity was weakly attributable to genetic contributions. However, if the shared environments are not accounted, the heritability of sebum, humidity increased to $0.41,0.38$ (arm), and 0.48 (cheek). It is noteworthy because some studies, such as nuclear family studies, cannot properly address shared environmental effects among family members. When shared environments are mixed with background polygenic effects, the heritability estimation might be inflated for skin sebum secretion or skin humidity levels.

The meaning of 'shared environments' for sebum secretion or humidity cannot be specified in this study alone. Because we adjusted for associated factors in each analysis, the 'common environments' refer to other unaddressed factors independent of both genetic or covariates in this study. Nutrition or other shared lifestyle factors, as well as indoor environments, might be possible explanations.

Quantitative genetic and environmental contributions to skin melanin index, sebum secretion, or humidity level would provide insight not only to the underlying biology of the traits, but related skin disease as well. For example, excessive sebum secretion or alterations in the composition of the secretion is a risk factor of acnes vulgaris. And low level of skin humidity is associated with eczematous dermatitis or pruritus (Freedberg et al., 2003). It is not clear with this study alone, whether genetic measures of skin melanin index in general healthy population are consistent with those among the clinical cases such as vitiligo. Our findings suggest that the role of sunlight exposure to skin melatonin index might be complex and probably interactive. The skin sites with more sunlight exposure (cheek) showed higher MZ ICC than with less (lower back skin). This might suggest that there exists a moderate level of heritability for baseline skin melatonin index, but the tanning ability might have more genetic influences.

This study confers several strengths. First, our data included various familial relationships in a Korean twin-family cohort, and these enabled us to decompose 
TABLE 3

Heritability $\left(h^{2}\right)$ Estimates of Skin Phenotypes

\begin{tabular}{|c|c|c|c|c|c|c|c|c|c|c|c|}
\hline \multirow[b]{2}{*}{ Skin phenotypes } & \multicolumn{5}{|c|}{$\mathrm{h}^{2} \pm S E[95 \% \mathrm{Cl}]$} & \multicolumn{4}{|c|}{ Variance component ${ }^{a}$} & \multirow[b]{2}{*}{$-2 L L^{b}$} & \multirow[b]{2}{*}{ Model } \\
\hline & Crude & $p$ value & $-2 \mathrm{LL}$ & Adjusted & $p$ value & A & C & D & $\mathrm{E}$ & & \\
\hline Melanin index & $\begin{array}{l}0.437 \pm 0.053 \\
{[0.384-0.490]}\end{array}$ & $<.0001$ & 18162.5 & $\begin{array}{l}0.486 \pm 0.049 \\
{[0.437-0.535]^{d}}\end{array}$ & $<.0001$ & 0.486 & 0.071 & - & 0.442 & 17996.7 & ACE \\
\hline \multirow[t]{2}{*}{ (flank) } & $\begin{array}{l}0.333 \pm 0.056 \\
{[0.277-0.389]}\end{array}$ & & 18142.8 & $\begin{array}{l}0.438 \pm 0.056 \\
{[0.382-0.494]}\end{array}$ & & 0.438 & - & 0.179 & 0.383 & 17993.0 & $A D E^{h}$ \\
\hline & $\begin{array}{l}0.547 \pm 0.033 \\
{[0.514-0.582]}\end{array}$ & $<.0001$ & 18171.6 & $\begin{array}{l}0.573 \pm 0.031 \\
{[0.542-0.604]}\end{array}$ & $<.0001$ & 0.573 & - & - & 0.427 & 18003.1 & $\mathrm{AE}$ \\
\hline Sebum & $\begin{array}{l}0.339 \pm 0.050 \\
{[0.289-0.389]}\end{array}$ & $<.0001$ & 1992.7 & $\begin{array}{l}0.211 \pm 0.049 \\
{[0.162-0.260]^{\mathrm{e}}}\end{array}$ & $<.0001$ & 0.211 & 0.158 & - & 0.632 & 1557.1 & $\mathrm{ACE}^{\mathrm{h}}$ \\
\hline \multirow[t]{2}{*}{ (glabella) } & $\begin{array}{l}0.439 \pm 0.051 \\
{[0.388-0.490]}\end{array}$ & & 1944.9 & $\begin{array}{l}0.410 \pm 0.033 \\
{[0.377-0.444]}\end{array}$ & $<.0001$ & 0.410 & - & 0.000 & 0.590 & 1590.2 & $A D E$ \\
\hline & $\begin{array}{l}0.457 \pm 0.035 \\
{[0.422-0.492]}\end{array}$ & $<.0001$ & 1945.2 & $\begin{array}{l}0.410 \pm 0.033 \\
{[0.377-0.444]}\end{array}$ & $<.0001$ & 0.410 & - & - & 0.590 & 1590.2 & $A E$ \\
\hline \multirow[t]{3}{*}{ Arm humidity } & $\begin{array}{l}0.147 \pm 0.054 \\
{[0.093-0.201]}\end{array}$ & .0023 & 1953.8 & $\begin{array}{l}0.125 \pm 0.055 \\
{[0.070-0.179]^{f}}\end{array}$ & .0088 & 0.125 & 0.188 & - & 0.688 & 1906.6 & $\mathrm{ACE}^{\mathrm{h}}$ \\
\hline & $\begin{array}{l}0.398 \pm 0.032 \\
{[0.366-0.430]}\end{array}$ & & & $\begin{array}{l}0.373 \pm 0.045 \\
{[0.329-0.418]}\end{array}$ & & 0.373 & - & 0.015 & 0.612 & 1940.8 & ADE \\
\hline & $\begin{array}{l}0.398 \pm 0.032 \\
{[0.366-0.430]}\end{array}$ & $<.0001$ & 1988.0 & $\begin{array}{l}0.381 \pm 0.032 \\
{[0.349-0.413]}\end{array}$ & $<.0001$ & 0.381 & - & - & 0.619 & 1940.9 & $\mathrm{AE}$ \\
\hline \multirow[t]{3}{*}{ Cheek humidity } & $\begin{array}{l}0.109 \pm 0.050 \\
{[0.059-0.159]}\end{array}$ & .0122 & 14821.8 & $\begin{array}{l}0.109 \pm 0.049 \\
{[0.060-0.158]^{9}}\end{array}$ & .0103 & 0.109 & 0.287 & - & 0.604 & 14700.7 & $\mathrm{ACE}^{\mathrm{h}}$ \\
\hline & $\begin{array}{l}0.443 \pm 0.045 \\
{[0.398-0.488]}\end{array}$ & & 14907.1 & $\begin{array}{l}0.469 \pm 0.044 \\
{[0.425-0.513]}\end{array}$ & & 0.469 & - & 0.023 & 0.507 & 14794.4 & ADE \\
\hline & $\begin{array}{l}0.474 \pm 0.031 \\
{[0.443-0.505]}\end{array}$ & $<.0001$ & 14908.1 & $\begin{array}{l}0.483 \pm 0.031 \\
{[0.452-0.514]}\end{array}$ & $<.0001$ & 0.483 & - & - & 0.517 & 14794.6 & $\mathrm{AE}$ \\
\hline
\end{tabular}

Note: ${ }^{a} \mathrm{~A}=$ additive genetics, $\mathrm{D}=$ dominant genetics, $\mathrm{C}=$ unmeasured shared environments, $\mathrm{E}=$ unique environments in the variance component model after adjusting for covariates; ${ }^{\mathrm{b}}-2 \times$ log-likelihood for the corresponding model after adjusting for covariates; ${ }^{\mathrm{c}} \mathrm{ACE}=$ additive genetics and unmeasured shared and unique environments included in the variance component model, ADE = additive and dominant genetics and unique environments included in the variance component mode, $\mathrm{AE}=$ additive genetics and unique environments included in the variance component model; ${ }^{\mathrm{d}}$ Adjusted for age, sex,

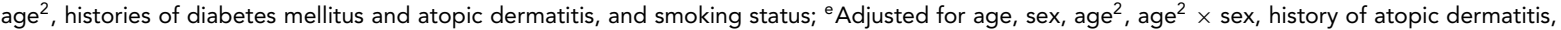
smoking status, and season; ${ }^{f}$ Adjusted for age, history of atopic dermatitis, and season; ${ }^{9}$ Adjusted for age, sex, history of hyperthyroidism, smoking status, and season; ${ }^{\text {h}}$ Best-fitting models shown in bold type were selected using likelihood ratio test.

genetics and environments of skin phenotypes more precisely than those from classical twin studies or conventional family studies. Second, traits were measured from relatively large number of subjects by trained staff in centralized clinics, using standardized protocols. This study reported genetic contributions to general skin phenotypes such as sebum and humidity. This study has some limitations as well. First, the nature of the skin traits may differ from the anatomical sites. Our findings may not represent the influences of genetics on other sites. Second, our findings are mainly focused on the first question of the ' 20 questions' about skin biology. Even though the melanocortin-1 receptor (MC1R) gene is known as one of major genes that determine skin pigmentation (Latreille et al., 2009), studies regarding which specific genetic variants are associated with skin melanin index in Asians or the environmental determinants of skin humidity will ensue. Third, the moderate genetic effects on the skin melanin index with adjustment for environments may need replications in other populations as well.

In summarizing, we estimated genetic and environmental contributions to commonly measured skin traits in a Korean twin-family cohort. Our findings suggest that level of inherited susceptibilities may differ between skin melanin index and sebum secretion or humidity level; genetics play a major role in skin melanin index but a lesser role in sebum secretion or humidity control. Sebum secretion and skin humidity are controlled predominantly by environmental factors, notably on shared environments among family members. We expect that our findings add insight to determinants of common dermatologic traits, and serve as a reference for biologic studies.

\section{Acknowledgments}

This work was supported by the National Project for Personalized Genomic Medicine, Ministry for Health \& Welfare, Republic of Korea (grant number A111218-12GM10); by the Basic Science Research Program through the National Research Foundation funded by the Ministry of Education, Science and Technology, Republic of Korea (J.S., grant numbers 2010-0025814, 2011-220-E00006), (Y.J.S., grant number 2015R1C1A2A01055129); by the Korea Healthcare Technology R\&D Project Ministry of Health and Welfare, Republic of Korea (J.S., Grant number A070001); and by Inha University Research grant (Y.J.S., grant number 50480).

\section{Disclosure of Interests}

None.

\section{Supplementary Material}

To view supplementary material for this article, please visit https://doi.org/10.1017/thg.2016.86. 


\section{References}

Abecasis, G. R., Cherny, S. S., Cookson, W. O., \& Cardon, L. R. (2002). Merlin-Rapid analysis of dense genetic maps using sparse gene flow trees. Nature Genetics, 30, 97-101.

Barsh, G. S. (2003). What controls variation in human skin color? PLoS Biology, 1, e27.

Bataille, V., Lens, M., \& Spector, T. D. (2012). The use of the twin model to investigate the genetics and epigenetics of skin diseases with genomic, transcriptomic and methylation data. Journal of the European Academy of Dermatology and Venereology, 26, 1067-1073.

Bataille, V., Snieder, H., MacGregor, A. J., Sasieni, P., \& Spector, T. D. (2002). The influence of genetics and environmental factors in the pathogenesis of acne: A twin study of acne in women. Journal of Investigative Dermatology, 119, 1317-1322.

Beasley, T. M., Erickson, S., \& Allison, D. B. (2009). Rankbased inverse normal transformations are increasingly used, but are they merited? Behavior Genetics, 39, 580-595.

Berardesca, E. (1997). EEMCO guidance for the assessment of stratum corneum hydration: Electrical methods. Skin Research and Technology, 3, 126-132.

Byard, P. J. (1981). Quantitative genetics of human skin color. American Journal of Physical Anthropology, 24, S123-S137.

Cho, E. B., Ha, J. M., Park, E. J., Kim, K. H., \& Kim, K. J. (2014). Heredity of acne in Korean patients. Journal of Dermatology, 41, 915-917.

Clark, P., Stark, A., Walsh, R., Jardine, R., \& Martin, N. (1981). A twin study of skin reflectance. Annals of Human Biology, $8,529-541$.

Freedberg, I. M., Eisen, A. Z., Wolff, K., Austen, K. F., Goldsmith, L. A., \& Katz, S. I. (2003). Fitzpatrick's dermatology in general medicine (vol. 51, 6th ed.). New York: McGraw-Hill.

Ginger, R. S., Blachford, S., Rowland, J., Rowson, M., \& Harding, C. R. (2005). Filaggrin repeat number polymorphism is associated with a dry skin phenotype. Archives of Dermatological Research, 297, 235-241.

Gombojav, B., Song, Y. M., Lee, K., Yang, S., Kho, M., Hwang, Y. C., Ko, G., \& Sung, J. (2013). The Healthy Twin Study, Korea updates: Resources for omics and genome epidemiology studies. Twin Research and Human Genetics, 16, 241-245.
Kang, H. Y. (2012). Melasma and aspects of pigmentary disorders in Asians. Annales de Dermatologie et de Venereologie, 139, S144-S147.

Latreille, J., Ezzedine, K., Elfakir, A., Ambroisine, L., Gardinier, S., Galan, P., ... Guinot, C. (2009). MC1R gene polymorphism affects skin color and phenotypic features related to sun sensitivity in a population of French adult women. Photochemistry and Photobiology, 85, 1451-1458.

Miyagaki, T., \& Sugaya, M. (2015). Recent advances in atopic dermatitis and psoriasis: Genetic background, barrier function, and therapeutic targets. Journal of Dermatological Science, 78, 89-94.

Paik, S. H., Kim, H. J., Son, H. Y., Lee, S., Im, S. W., Ju, Y. S., ... Kim, J. I. (2012). Gene mapping study for constitutive skin color in an isolated Mongolian population. Experimental \& Molecular Medicine, 44, 241-249.

Paternoster, L., Standl, M., Chen, C.-M., Ramasamy, A., Bønnelykke, K., Duijts, L., ... Albrecht, E. (2012). Metaanalysis of genome-wide association studies identifies three new risk loci for atopic dermatitis. Nature Genetics, 44, 187192.

Pierard, G. E. (1998). EEMCO guidance for the assessment of skin colour. Journal of the European Academy of Dermatology and Venereology, 10, 1-11.

Pierard, G. E., Pierard-Franchimont, C., Marks, R., Paye, M., \& Rogiers, V. (2000). EEMCO guidance for the in vivo assessment of skin greasiness. The EEMCO Group. Skin Pharmacology and Applied Skin Physiology, 13, 372-389.

Pons-Guiraud, A. (2007). Dry skin in dermatology: A complex physiopathology. Journal of the European Academy of Dermatology and Venereology, 21, S1-S4.

Song, Y. M., Lee, D., Lee, M. K., Lee, K., Lee, H. J., Hong, E. J., Han, B., \& Sung, J. (2010). Validity of the zygosity questionnaire and characteristics of zygosity-misdiagnosed twin pairs in the Healthy Twin Study of Korea. Twin Research and Human Genetics, 13, 223-230.

Sung, J., Cho, S. I., Lee, K., Ha, M., Choi, E. Y., Choi, J. S., ... Song, Y. M. (2006). Healthy Twin: A twin-family study of Korea - Protocols and current status. Twin Research and Human Genetics, 9, 844-848.

Walton, S., Wyatt, E. H., \& Cunliffe, W. J. (1988). Genetic control of sebum excretion and acne - A twin study. British Journal of Dermatology, 118, 393-396. 